\title{
Exogenous Abscisic Acid and Gibberellic Acid Elicit Opposing Effects on Fusarium graminearum Infection in Wheat
}

\author{
Leann M. Buhrow, Dustin Cram, Dan Tulpan, Nora A. Foroud, and Michele C. Loewen
}

First, second, and fifth authors: National Research Council of Canada, Aquatic and Crop Resources Development, 110 Gymnasium Place, Saskatoon, SK, S7N 0W9 Canada; third author: National Research Council of Canada, Information and Communication Technologies, 100 des Aboiteaux Street, Moncton, NB, E1A 7R1 Canada; fourth author: Agriculture and Agri-Food Canada, 5403 1st Ave S., Lethbridge, AB, T1J 4B1 Canada; and fifth author: Department of Biochemistry, University of Saskatchewan, 107 Wiggins Rd. Saskatoon, SK, S7N 5E5 Canada.

Accepted for publication 18 April 2016.

\begin{abstract}
Buhrow, L. M., Cram, D., Tulpan, D., Foroud, N. A., and Loewen, M. C. 2016. Exogenous abscisic acid and gibberellic acid elicit opposing effects on Fusarium graminearum infection in wheat. Phytopathology 106:986-996.

Although the roles of salicylate (SA) and jasmonic acid (JA) have been well-characterized in Fusarium head blight (FHB)-infected cereals, the roles of other phytohormones remain more ambiguous. Here, the association between an array of phytohormones and FHB pathogenesis in wheat is investigated. Comprehensive profiling of endogenous hormones demonstrated altered cytokinin, gibberellic acid (GA), and JA metabolism in a FHB-resistant cultivar, whereas challenge by Fusarium graminearum increased abscisic acid (ABA), JA, and SA in both FHB-susceptible and

These phytohormones-induced effects may be attributed to alteration of the $F$. graminearum transcriptome because ABA promoted expression of earlyinfection genes, including hydrolases and cytoskeletal reorganization genes, while GA suppressed nitrogen metabolic gene expression. Neither ABA nor GA elicited significant effects on $F$. graminearum fungal growth or sporulation in axenic conditions, nor do these phytohormones affect trichothecene gene expression, deoxynivalenol mycotoxin accumulation, or SA/JA biosynthesis in F. graminearum-challenged wheat spikes. Finally, the combined application of GA and paclobutrazol, a Fusarium fungicide, provided additive effects on reducing FHB severity, highlighting the potential for combining fungicidal agents with select phytohormone-related treatments for management of FHB infection in wheat.
\end{abstract} -resistant cultivars. Subsequent investigation of ABA or GA coapplication with fungal challenge increased and decreased FHB spread, respectively.

Fusarium head blight (FHB) is a devastating disease of wheat and other small grain cereals caused by species in the Fusarium genus, with the most prevalent species being Fusarium graminearum and F. culmorum (Edwards 2004; Parry et al. 1995). F. graminearum's hemibiotrophic lifestyle involves establishment on and penetration into its host, where optimal fitness and virulence is dependent on nitrogen metabolism (Divon et al. 2006; Namiki et al. 2001; Seong et al. 2005) and biosynthesis of virulence factors such as the trichothecene mycotoxin deoxynivalenol (DON) (Gale 2003; Kang and Buchenauer 1999, 2000; McCormick 2003). DON is not only phytotoxic, reducing grain quality and yield, but also poses a threat to human and animal health when consumed (Antonissen et al. 2014; Mostrom and Raisbeck 2007). FHB is manifested as bleaching or necrotic browning of spike tissue, ultimately producing shriveled or discolored grain (McMullen et al. 1997). In spite of extensive wheat breeding efforts, only a limited number of FHB-resistant cultivars have been developed, where 'Sumai 3' and its derivatives exhibit the greatest resistance (Bai and Shaner 2004). The use of chemical fungicides has also been investigated; however, these reports highlight the inconsistency and reduced efficacy of available fungicides in FHB-susceptible wheat varieties (Mesterházy et al. 2003).

Phytohormones commonly associated with plant defense against pathogens, including salicylic acid (SA) and jasmonic acid (JA)

Corresponding author: M. C. Loewen; E-mail address: michele.loewen@nrc.ca

*The $e$-Xtra logo stands for "electronic extra" and indicates that four supplementary figures and three supplementary tables are published online.

http://dx.doi.org/10.1094/PHYTO-01-16-0033-R

(C) 2016 The American Phytopathological Society
Additional keywords: grain yield.
(De Vleesschauwer et al. 2014), have recently been investigated to elucidate their roles in FHB resistance. Both SA and JA have been reported to possess antifungal activity against $F$. graminearum (Qi et al. 2012, 2016; Sun et al. in press) and also mediate induced defense responses in planta through their signaling activities (Makandar et al. 2006; Qi et al. 2016; Sun et al. in press). In fact, independent studies have demonstrated that overexpression of an SA-signaling gene in the otherwise susceptible wheat 'Bobwhite' is sufficient to promote FHB resistance (Makandar et al. 2006), and related SA-signaling genes may serve as markers for resistance in a diverse set of winter wheat cultivars (Diethelm et al. 2014). Although SA and JA signaling have been reported to contribute to FHB resistance, the role of JA has also been reported to contribute to FHB susceptibility, specifically during the early stages of infection (Ameye et al. 2015; Ding et al. 2011; Makandar et al. 2010, 2012). Together, these studies suggest an infection model where SA promotes resistance during early $F$. graminearum biotrophic growth, whereas JA promotes resistance only in the later necrotrophic infection (Ameye et al. 2015; Ding et al. 2011; Makandar et al. 2010, 2012).

Although the roles of SA and JA signaling in the FHB disease response have been well characterized, the role of other phytohormones remains more ambiguous. By combining differential gene expression and exogenous hormone application, ethylene (ET) has been reported to be associated with FHB resistance (Li and Yen 2008), FHB susceptibility (Chen et al. 2009), or have no effect on pathogenesis (Sun et al. in press). In addition, isolated studies of exogenous auxin (the auxin indole 3 -acetic acid [IAA] was used throughout the study) or epibrassinolied application immediately prior to Fusarium infection have been reported to reduce FHB disease severity and yield loss (Ali et al. 2013; Petti et al. 2012). Finally, gibberellic acids (GA) have been reported to induce 
pathogen-resistance-associated gene expression (Casacuberta et al. 1992) and promote resistance to ascomycota fungal infections (Eshel et al. 2002; Tanaka et al. 2006), though the effect of GA has not previously been investigated in relation to FHB. The reports in this field to date have also suggested a complex interaction among phytohormones in the FHB response, potentially involving crosstalk between SA, JA, IAA, and abscisic acid (ABA) signaling pathways (Qi et al. 2016; Yang et al. 2015).

The work described herein focused on elucidating the roles of ABA, GA, and other less-well-characterized hormones in the FHB-wheat interaction. Unique associations between phytohormones and FHB resistance were identified by evaluating endogenous and Fusarium-induced phytohormone profiles in FHB-resistant (Sumai 3) and FHB-susceptible ('Fielder') Triticum aestivum wheat cultivars. Exogenous coapplication of diverse phytohormones was subsequently investigated and included independent application of ABA, IAA, GA, and the cytokinin zeatin (Z). Effects of these phytohormones on axenic $F$. graminearum growth and sporulation as well as FHB spread, DON biosynthesis and accumulation, and hormonal crosstalk in $F$. graminearum-challenged wheat spikes are evaluated. Finally, the effects of applying phytohormone analogs and combining phytohormones and fungicide treatments are also discussed. This work highlights the potential value of combining phytohormone-related treatments with existing fungicidal applications as part of an agronomic strategy for mitigating FHB pathogenesis in wheat.

\section{MATERIALS AND METHODS}

Chemicals and phytohormones. IAA, GA A3, racemic Z, and paclobutrazol (PBZ) were purchased from Sigma-Aldrich (St. Louis). The National Research Council Hormone Profiling Facility provided (+)-ABA while 3'-hexasulfanyl-(+)-ABA was synthesized as described (Takeuchi et al. 2014) and provided by Kenneth Nelson and Suzanne Abrams at the University of Saskatchewan. ABA and its chemical analogs were assessed by nuclear magnetic resonance prior to acquisition. Phytohormone stocks were solubilized in deionized water as sodium salts by $1.0 \mathrm{~N} \mathrm{NaOH}$ titration and stored at $-20^{\circ} \mathrm{C}$ in amber vials. Working solutions were made in deionized water and $\mathrm{pH}$ was adjusted, as needed, to 7.0 with $0.1 \mathrm{~N} \mathrm{HCl}$. PBZ (and GA for direct comparison) stocks were solubilized in $50 \%$ ( $\mathrm{vol} / \mathrm{vol}$ ) methanol and working solutions were made by dilution in deionized water, with the final solution having no more than $10 \%$ (vol/vol) methanol. Chemdraw Pro v11 (CambridgeSoft, Waltham, MA) was used to depict chemical structures.

F. graminearum inoculum preparation. $F$. graminearum GZ3639 (Proctor et al. 1995) was propagated on potato dextrose agar (PDA; Sigma-Aldrich) at $25^{\circ} \mathrm{C}$ for 5 days. To obtain spores, carboxymethylcellulose liquid media (CMC; Sigma-Aldrich) was inoculated with a marginal 5-mm-square PDA plug and grown for 5 days at $27^{\circ} \mathrm{C}$, with shaking at $180 \mathrm{rpm}$. Spores were isolated by filtering through one layer of cheesecloth and 25- $\mu \mathrm{m}$ Miracloth filter (EMD Millipore, Billerica, MA), washed three times with sterile water, and quantified using a hemocytometer and light microscopy.

F. graminearum growth and sporulation quantification. The effects of ABA, IAA, GA, Z, PBZ, or the respective solvent (deionized water or $10 \%$ [vol/vol] methanol) were evaluated for their ability to alter Fusarium spp. growth and sporulation. PDA plates were supplemented with $1.0 \mathrm{mM}$ ABA, IAA, GA, or Z or $100 \mu \mathrm{M}$ PBZ or solvent controls and inoculated with marginal 5-mm-square PDA plugs or 500 isolated $F$. graminearum spores. Radial growth was monitored in triplicate as the average of three diameter measurements, at approximately $45^{\circ}$ angles, over the course of seven consecutive days. Potato dextrose broth (SigmaAldrich) consisting of 100-ml cultures supplemented with $1.0 \mathrm{mM}$ ABA, IAA, GA, or Z or $100 \mu \mathrm{M}$ PBZ were inoculated with 500 isolated spores and shaken at $180 \mathrm{rpm}$ and $27^{\circ} \mathrm{C}$ for 7 days. Fungal mass was measured in triplicate after vacuum filtration through $11-\mu \mathrm{m}$ filter paper and lyophilization. CMC 50-ml cultures supplemented with $1.0 \mathrm{mM}$ ABA, IAA, GA, or Z or $100 \mu \mathrm{M}$ PBZ were inoculated with marginal 5-mm-square PDA plugs and shaken at $180 \mathrm{rpm}$ and $27^{\circ} \mathrm{C}$ for 5 days. Fusarium spp. sporulation was quantified in triplicate as the number of spores isolated after successive cheesecloth and 25- $\mu \mathrm{m}$ Miracloth filtering per milliliter of culture.

Propagation of plants and grain preparation. T. aestivum Fielder and Sumai 3 were grown in Sunshine Mix 8 (Sungrow Horticulture, Agawam, MA) and maintained in climate-controlled chambers with a 16 -photoperiod at $25^{\circ} \mathrm{C}$ followed by $8 \mathrm{~h}$ of darkness at $16^{\circ} \mathrm{C}$ every day. Plants were watered as needed and fertilized biweekly with 20-20-20 N-P-K. At the two-leaf stage, plants were treated with Intercept (Bayer Crop Science, Calgary, AB, Canada) as a powdery mildew and aphid preventative. For preparation of grain, wheat was allowed to mature naturally under normal growing conditions and grain was manually isolated.

Phytohormone-only application to Fielder spikes for phenotypic assessment. Fielder spikes were treated at each spikelet with $10 \mu \mathrm{l}$ of $1.0 \mathrm{mM}$ ABA, IAA, GA, Z, or deionized water (mock) during anthesis. Wheat was incubated in climate-controlled conditions as described above until spikes naturally matured. Grain from each treatment was manually isolated, where spikes were dried and grain was harvested from each spikelet using a forceps, including the small Fusarium-damaged kernels. The resulting samples were characterized for yield and germination, as described below.

Fusarium challenge of Fielder and Sumai 3 spikes. During anthesis, two florets from a central spikelet were point inoculated with $10 \mu \mathrm{l}$ of a suspension of $5.0 \times 10^{4} \mathrm{~F}$. graminearum GZ3639 spores or deionized water (mock). To promote infection, wheat plants were transferred to climate-controlled chambers with $90 \%$ humidity for $72 \mathrm{~h}$, with the same light and temperature conditions as described above, then returned to ambient humidity. Challenged spikes were monitored for phenotypic evidence of FHB in each spikelet of all inoculated spikes for 14 days. Spike and grain tissue were harvested from $F$. graminearum-challenged and unchallenged treatment groups, where individual spikes were independently processed while grain samples were prepared by allowing spikes to dry and then pooling harvested grain within a treatment group into 1.0-g biological replicate samples. Tissues were analyzed for phytohormone content, DON contamination, and yield.

Hormone profiling of Fusarium-challenged Fielder and Sumai 3 spikes. Fielder and Sumai 3 spikes were $F$. graminearum challenged as described above. Fourteen days postchallenge, five biological replicates, consisting of one individual spike each of mock-inoculated Fielder, Fusarium-inoculated Fielder, mock-inoculated Sumai 3, and Fusarium-inoculated Sumai 3, were flash frozen and ground in liquid nitrogen. Phytohormones were extracted from individual replicate spikes and quantified by ultrahigh-performance liquid chromatography electrospray ionization (ESI) tandem mass spectrometry at the National Research Council of Canada, Aquatic and Crop Resources Development Portfolio, as described (Abrams et al. 2003; Galka et al. 2005; Lulsdorf et al. 2013; Ross et al. 2004; Zaharia et al. 2005).

DON quantification in Fusarium-infected Fielder spikes and grain. Fielder spikes were $F$. graminearum challenged as described earlier, and spikes and grain were sampled. In particular, 10 spikes, with each spike representing one biological replicate, were analyzed individually at the indicated times postchallenge. For grain sampling, remaining plants were allowed to mature and all grain from each treatment group was isolated using forceps and pooled. This pooled grain was mixed, and three 1.0-g replicates were made from the pooled, mixed total sample. In both instances, each individual sample was ground under liquid nitrogen and solvent extracted in five volumes of $84 \%$ ( $\mathrm{vol} / \mathrm{vol}$ ) acetonitrile by shaking at $220 \mathrm{rpm}$ and $25^{\circ} \mathrm{C}$ for $2 \mathrm{~h}$. Extracted DON was quantified relative to a commercial standard (Sigma-Aldrich) by liquid chromatography-mass spectrometry (LC-MS) modified from Plattner and Maragos (2003). Briefly, DON was separated from 
coextracted molecules with a Waters 2695 LC coupled with a Waters Symmetry C 18 column ( 100 by $2.1 \mathrm{~mm}$ i.d., $3.5 \mu \mathrm{m})$ at $25^{\circ} \mathrm{C}$. The mobile phase was composed of $0.3 \%$ (vol/vol) acetic acid (A) and $95 \%$ (vol/vol) methanol (B); A under gradient elusions at 0 to $7 \mathrm{~min}$; $99 \% \mathrm{~A}, 7$ to $25 \mathrm{~min} ; 67 \% \mathrm{~A}$ and $33 \% \mathrm{~B}, 25$ to $30 \mathrm{~min}$; and $99 \%$ A. MS analysis was performed using a Waters 3100 Mass Detector fitted with ESI in negative ion mode and an optimized $40-\mathrm{V}$ cone voltage. DON was detected at $\mathrm{m} / z$ of $355.3 \mathrm{Da}$ and analyzed using Empower Pro Software (Waters, Milford, MA).

Grain yield and germination determination. Fielder spikes were phytohormones treated or $F$. graminearum challenged, as described above. Thousand-grain weight was selected as the yield metric and calculated, in triplicate, as five times the mass of 200 grains randomly selected from the pooled grain per treatment group, as described previously (Pask et al. 2012). For germination studies, triplicate groups of 20 seeds from each treatment group were washed with a $5 \%$ ( $\mathrm{vol} / \mathrm{vol})$ hypochlorite solution, imbibed in deionized water, and incubated in the dark at $25^{\circ} \mathrm{C}$ for 10 days. Germination was evaluated by the visual presence of a coleorhiza.

Coapplication of phytohormones with Fusarium challenge to Fielder spikes. Fielder spikes were Fusarium challenged as described earlier but the inoculum was supplemented with hormones or PBZ. For coinoculation with hormones, compounds were solubilized in deionized water and a total of six treatments were assessed: $1.0 \mathrm{mM}$ ABA, IAA, GA, Z, or AS6, or deionized water (mock). Each coinoculum was applied to a minimum of 30 spikes during anthesis, where two florets from a central spikelet were inoculated, and repeated in triplicate. For coinoculation with PBZ, compounds were solubilized in $10 \%(\mathrm{vol} / \mathrm{vol})$ methanol and a total of four treatments were assessed: $1.0 \mathrm{mM}$ GA, $10 \mu \mathrm{M}$ PBZ, $10 \mu \mathrm{M}$ PBZ plus $1.0 \mathrm{mM}$ GA, or $10 \%$ (vol/vol) methanol (mock). For PBZ coinoculations, each treatment was applied to a minimum of 15 spikes and repeated in triplicate. In both coapplication experiments, inoculated spikes were rated for the number of diseased spikelets over a 14-day period. Seven days postinoculation, five spikes (biological replicates) from the $\mathrm{ABA}, \mathrm{GA}$, and mock treatment groups were analyzed for phytohormone content as described above. Fourteen days after inoculation, 10 spikes (biological replicates) from each treatment were analyzed for DON contamination, with the exception of the PBZ coapplication experiments, where only 5 spikes were analyzed for DON content. The remaining wheat was allowed to mature and grain from each treatment was characterized for yield and DON contamination, as described above.

RNA sequencing and $\boldsymbol{F}$. graminearum expression analysis. Total RNA was extracted from five Fielder spikelets (biological replicates), each challenged with $F$. graminearum in the presence and absence of $1.0 \mathrm{mM} \mathrm{ABA}$ or GA, at $24 \mathrm{~h}$ and 5 days postchallenge. RNA was purified using the RNeasy Plant Mini Kit (Qiagen, Mississauga, CA) and treated with DNaseI (Qiagen) according to the manufacturer's instructions. RNA quantity and quality were evaluated using NanoDrop ND-8000 (NanoDrop, Wilmington, DE) and agarose gel electrophoresis.

Total RNA isolated from Fielder spikelets $24 \mathrm{~h}$ after being challenged with $F$. graminearum in the presence and absence of 1.0 mM ABA or GA were sequenced. RNA library construction and HiSeq RNA sequencing were performed at the National Research Council of Canada DNA Sequencing Facility (Saskatoon, SK, Canada). RNA libraries were prepared using $1.0 \mu \mathrm{g}$ of total RNA and the TruSeq RNA Sample Prep Kit v2 (Illumina, San Diego, CA) according to the manufacturer's instructions. Library quality was assessed on the 2100 Bioanalyzer (Agilent Technologies Inc., Santa Clara, CA) equipped with a DNA 1000 chip. Library concentrations were determined by quantitative polymerase chain reaction (qPCR) using the KAPA SYBR FAST ABI Prism qPCR Kit (Kapa Biosystems, Wilmington, MA) and the StepOnePlus Real-Time PCR System (Applied Biosystems, Foster City, CA). RNA samples were multiplexed at a sequencing depth of five libraries per lane. Equimolar concentrations of the libraries were pooled and a final concentration of $12 \mathrm{pM}$ was used for clustering in cBOT (Illumina) flowcell lanes. The samples were then sequenced ( $2 \times 101$ cycles, paired-end reads) on the HiSeq2500 (Illumina) using the TruSeq SBS Kit v3-HS 200 cycles Kit (Illumina). Raw RNA sequence reads were processed with Trimmomatic v0.32 (Bolger et al. 2014) to remove Truseq 3 adaptor sequences, lowquality bases selected using five-base averaging and a quality threshold of 20, and reads with fewer than 50 bases. The trimmed reads were mapped against the $F$. graminearum $\mathrm{PH}-1$ (FG3) genome (Fusarium Comparative Sequencing Project, Broad Institute of Harvard and MIT) (http://www.broadinstitute.org/) using STAR v2.4.0j (Dobin et al. 2012). Per sample GTF files were merged using cuffmerge from the Cufflinks v2.1.1 suite (Trapnell et al. 2010). Read counts were calculated using HTSEquation 0.6.0 (Anders et al. 2015) htseq-count in unstranded mode. Comparisons between individual treatments and control were evaluated using the DESeq2 v1.6.3 (Love et al. 2014) Wald statistic. F. graminearum genes were annotated using information extracted from two public sources: (i) the Protein Extraction, Description and Analysis Tool version 3 (PEDANT 3) from the Munich Information Center for Protein Sequences and (ii) the MycoCosm tool from the Joint Genome Institute (Cuomo et al. 2007), which hosts an annotated copy of the genome of $F$. graminearum strain PH-1 (NRRL 31084) sequenced by the Broad Institute. The RNASeq data generated by this work is available at the National Center for Biotechnology Information SRA under BioSample accession numbers SAMN04386757, SAMN04386758, and SAMN04386759 for $F$. graminearum, $F$. graminearum $+\mathrm{GA}$ and $F$. graminearum $+\mathrm{ABA}$ treatments, respectively.

Relative $\boldsymbol{F}$. graminearum gene expression determined by semiquantitative reverse-transcription PCR. Total RNA isolated from Fielder spikelets 5 days after being challenged with $F$. graminearum in the presence and absence of $1.0 \mathrm{mM} \mathrm{ABA}$ and GA were selected for relative gene expression analysis. cDNA was synthesized from $0.5 \mu \mathrm{g}$ of RNA using the Superscript III reversetranscription kit (Invitrogen, Carlsbad, CA). F. graminearum actin (GenBank XM_011328784.1) and trichothecene biosynthetic cluster genes (Kulik et al. 2012) tri4 (GenBank: AAK33083.1), tri5 (GenBank: AAK33084.1), and tri11 (GenBank: AAK33080.1) were compared with the wheat heterogeneous nuclear ribonucleoprotein Q ( $h n-P N P-Q$, Ta.10105) (Qi et al. 2012) reference gene using the comparative cycle threshold method (Livak and Schmittgen 2001). StepOne Plus Real-Time PCR System (Applied Biosystems, Foster City, CA) and StepOne Software v2.3 (Thermo Fisher Scientific Inc., Carlsbad, CA) were used to quantify and analyze relative expression. All primers in this work were designed using Primer3 v0.4.0 (Untergasser et al. 2012) and are listed in Supplementary Table S3.

Statistical analysis. Phytohormone content differences in Fielder and Sumai 3 with and without Fusarium challenging were analyzed with two-way analysis of variance (ANOVA) with Sidak posthoc comparisons. Unless otherwise noted, all other data (Fusarium spp. growth and sporulation, grain germination, spikelet infection, DON contamination, yield, and relative gene expression) were analyzed with one-way ANOVA with Dunnett posthoc comparisons. Comparisons were performed with GraphPad Prism 6 (GraphPad Software, Inc., La Jolla, CA).

\section{RESULTS}

Hormone profiling of susceptible and resistant wheat cultivars in $\boldsymbol{F}$. graminearum-challenged and unchallenged spikes. Phytohormone signaling and metabolism have been well characterized in a variety of wheat lines shortly after Fusarium spp. challenge, with these studies supporting an infection model where SA promotes resistance during early $F$. graminearum growth, while JA promotes resistance only in the necrotrophic infection (Ameye et al. 2015; Ding et al. 2011; Makandar et al. 2006, 2010, 2012; 
Qi et al. 2012, 2016; Sun et al. in press). Toward detecting possible involvement of these classical hormones in responses at later stages in FHB disease progression, as well as any roles for the nonclassical hormones (Supplementary Fig. S1), constitutive and Fusariuminduced hormonal changes were compared between the susceptible and resistant T. aestivum wheat Fielder and Sumai 3, respectively, at 14 days postinfection. This time point was selected to allow FHB to spread from the site of $F$. graminearum challenge to the adjacent tissue along the spike. Interestingly, in unchallenged cultivars, the resistant Sumai 3 demonstrated at least twofold higher levels of $\mathrm{ABA}$ and its metabolites 7'-OH $\mathrm{ABA}$ and $\mathrm{ABA}$ glucose ester, as well as SA, JA, and JA-isoleucine compared with the FHBsusceptible Fielder (Table 1). Sumai 3 also exhibited higher cytokinin metabolism based on the 20-fold lower levels of the cytokinin biosynthetic precursor Z-riboside and higher content of the cytokinin metabolite Z-O-glucoside. Finally, Sumai 3 displayed higher accumulation of the bioactive GA1 biosynthetic precursor GA19 and no detectable bioactive GA4 biosynthetic precursor GA24 compared with Fielder, suggesting that different endogenous GA metabolism pathways may be functioning in these cultivars (Yamaguchi 2008). Levels of other phytohormones and their metabolites, including IAA, did not differ between the unchallenged cultivars.

Toward investigating the effect of FHB on these profiles, FHBresistant Sumai 3 and FHB-susceptible Fielder spikes were challenged with $F$. graminearum spores by point inoculation, and phytohormone responses were quantified. Fourteen days after $F$. graminearum challenge, ABA and JA as well as their respective metabolites were increased by more than 50 and $200 \%$, respectively, while conjugated SA levels were depleted by approximately 50\% compared with unchallenged Fielder spikes (Table 1). Similar responses in the SA and ABA metabolic pathways were observed in Sumai 3, whereas no changes in JA or related metabolites were observed in this line. The absence of a Fusarium spp.induced response in JA and related metabolites may be due to the JA biosynthetic pathway being activated in Sumai 3 prior to Fusarium challenge or constitutively greater JA pools accumulating in this FHB-resistant cultivar. Ultimately, the intrinsic differences in phytohormone profiles between Fielder and Sumai 3 cultivars may be due to physical or developmental differences inherent to each line. However, these differences may also provide insight into how these cultivars respond to biotic stresses such as FHB. Consensus between phytohormone profiles, signaling, and effects of exogenous applications provide complementary approaches to describe the fundamental roles of phytohormones in response to pathogens.

Coapplication of exogenous ABA or GA promotes and reduces FHB symptoms, respectively, in FHB-susceptible Fielder. Based on the findings that FHB-susceptible and -resistant cultivars have unique phytohormone profiles and that these phytohormone profiles are responsive to Fusarium challenge, the direct effect of phytohormones on FHB disease symptoms and mycotoxin accumulation were evaluated in susceptible Fielder spikes. Phytohormones $\mathrm{ABA}, \mathrm{GA}, \mathrm{IAA}$, and $\mathrm{Z}$ were coinoculated with purified $F$. graminearum spores, while SA and JA were not included in this study because the effects of their application to wheat heads have been previously reported elsewhere (Qi et al. 2012; Qi et al. 2016; Makandar et al. 2012). FHB spread exhibited a degree of variability between independent trials and, as such, was represented as the average of three experiments. Coapplication of $1.0 \mathrm{mM}$ ABA increased the rate of FHB spread, especially from symptomatic tissue to an adjacent asymptomatic spikelets (Fig. 1), in a statistically significant manner throughout at least one-third of the challenged Fielder spike (Supplementary Fig. S2). Conversely, coapplication of $1.0 \mathrm{mM}$ GA reduced the spread of FHB most significantly from 7 to 10 days after Fusarium challenge (Fig. 1). This single application of GA was not sufficient to prevent disease spread; however, the delay in spread manifested itself in beneficial grain attributes, including reduced DON contamination and increased yield (Fig. 1B and C). ABA did not have a significant effect on either grain quality or quantity. Neither IAA nor Z elicited any significant changes in FHB response or DON accumulation in either spikes or grain (Supplementary Fig. S3).

The reduction of FHB disease symptoms in Fielder spikes treated with GA was consistent with reduced detection of $F$. graminearum gene expression in challenged spikelets. Two florets from a central spikelet were coinoculated with $F$. graminearum spores supplemented with either $1.0 \mathrm{mM} \mathrm{ABA}$ or GA, and the abundance of $F$. graminearum was monitored based on the expression of its

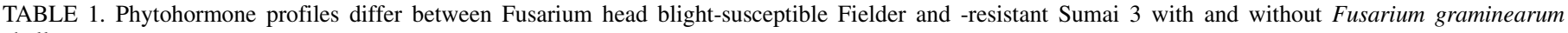
challenge $^{\mathrm{a}}$

\begin{tabular}{|c|c|c|c|c|}
\hline \multirow[b]{2}{*}{ Phytohormones $(\mathrm{ng} / \mathrm{g})^{\mathrm{b}}$} & \multicolumn{2}{|c|}{ Mock } & \multicolumn{2}{|c|}{ Fusarium challenged } \\
\hline & Fielder & Sumai 3 & Fielder & Sumai 3 \\
\hline $\mathrm{ABA}$ & $150 \pm 31$ & $280 \pm 97+$ & $260 \pm 77$ & $420 \pm 73 *+\dagger$ \\
\hline 7'-OH ABA & $8.4 \pm 2.9$ & $75 \pm 37+t+1$ & $14 \pm 6.6$ & $85 \pm 5.5 \dagger \dagger$ \\
\hline ABA glucose ester & $18 \pm 15$ & $98 \pm 71+t$ & $26 \pm 23$ & $67 \pm 19$ \\
\hline Dihydrophaseic acid & $28 \pm 10$ & $20 \pm 3.5$ & $71 \pm 65$ & $38 \pm 12$ \\
\hline Phaseic acids & $130 \pm 34$ & $51 \pm 23$ & $210 \pm 98 *$ & $82 \pm 19+\dagger$ \\
\hline Total ABA catabolites & $180 \pm 62$ & $240 \pm 130$ & $460 \pm 190 * *$ & $270 \pm 56$ \\
\hline cis-Zeatin-riboside & $150 \pm 36$ & $3.8 \pm 1.1+t+\dagger$ & $112 \pm 12 *$ & $5.2 \pm 0.80+\dagger+\dagger$ \\
\hline cis-Zeatin & $2.4 \pm 1.2$ & ND & $3.8 \pm 3.5$ & $1.5 \pm 0.30$ \\
\hline Trans-Zeatin & ND & $2.2 \pm 0.73+t+t$ & ND & $2.4 \pm 0.76+t+\dagger$ \\
\hline cis-Zeatin- $O$-glucoside & $94 \pm 18$ & $180 \pm 32+t+\dagger$ & $79 \pm 17$ & $130 \pm 10 * *+\dagger$ \\
\hline Trans-Zeatin- $O$-glucoside & $41 \pm 19$ & $110 \pm 46+t \dagger$ & $46 \pm 8.7$ & $81 \pm 11$ \\
\hline IAA & $4,320 \pm 1,090$ & $5,160 \pm 1,670$ & $4,090 \pm 1,270$ & $6,280 \pm 3,190$ \\
\hline IAA-aspartate & $129 \pm 244$ & $40 \pm 18$ & $2,400 \pm 3,170$ & $2,860 \pm 3,210$ \\
\hline GA19 & $27 \pm 7$ & $49 \pm 16 \dagger$ & $30 \pm 8$ & $38 \pm 19$ \\
\hline GA24 & $14 \pm 7$ & ND+t+ & $8 \pm 2$ & $\mathrm{ND}+$ \\
\hline GA44 & $12 \pm 2$ & $9 \pm 3$ & $12 \pm 4$ & $5 \pm 2+\dagger$ \\
\hline SA & $122 \pm 32$ & $245 \pm 61+t$ & $137 \pm 56$ & $195 \pm 27$ \\
\hline Conjugated SA & $1120 \pm 77$ & $1200 \pm 219$ & $682 \pm 48 * * *$ & $880 \pm 102^{* *}$ \\
\hline JA & $23 \pm 3$ & $67 \pm 17+t+t$ & $64 \pm 12 * * * *$ & $71 \pm 10$ \\
\hline JA-isoleucine & $11 \pm 5$ & $21 \pm 7+\dagger$ & $20 \pm 4^{*}$ & $18 \pm 3$ \\
\hline
\end{tabular}

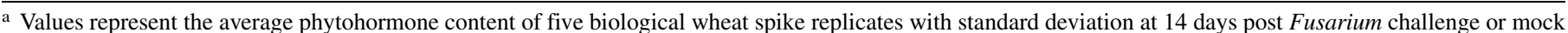
inoculation. Phytohormones that were not detected in three or more samples are noted as ND. Phytohormone differences between wheat cultivars $(\dagger, \dagger+, \dagger+$, and $+\dagger+$ indicate $P \leq 0.05,0.01,0.001$, and 0.0001 , respectively) and changes induced upon Fusarium challenge inoculation (*, **, ***, and **** indicate $P \leq 0.05$, $0.01,0.001$, and 0.0001 , respectively) were evaluated by two-way analysis of variance with Sidak posthoc comparisons.

b Abbreviations: abscisic acid (ABA), auxin (IAA), gibberellic acid (GA), and jasmonic acid (JA). 
constitutively expressed actin gene relative to the wheat $R N P Q$ reference gene (Qi et al. 2012) in the challenged spikelet and the adjacent basipetal node. Although actin has been widely reported as a housekeeping gene, the possibility of nonspecific semiquantitative reverse-transcription (RT)-PCR amplification as well as actin expression changes during the course of infection cannot be ignored. To this end, three additional genes encoded by F. graminearum but not wheat were also quantified: trichothecene biosynthetic cluster genes tri4, tri5, and tri11. Five days after Fusarium challenge, just prior to the phenotypic observation of FHB disease spread, the application of GA was found to consistently reduce the levels of detectable $F$. graminearum actin and tri gene expression in Fielder spike tissue (Fig. 2A), whereas ABA application had no significant effect. This reduced gene expression, in addition to the corresponding reduced
FHB disease symptoms (Fig. 1), is consistent with reduced Fusarium fungal spread upon GA coapplication.

Coapplication of ABA or GA does not affect trichothecene biosynthetic gene expression or DON accumulation in Fusarium spp.-challenged Fielder spikes. The virulence of $F$. graminearum is dependent on its ability to biosynthesize trichothecene mycotoxins, including DON. Coapplication studies demonstrated that, in addition to modulating FHB phenotypic symptoms, GA also affects the accumulation of DON in isolated grains (Fig. 1). This DON accumulation may either be a correlative symptom of limiting FHB spread or a direct effect on DON biosynthesis or accumulation. Five days after Fusarium challenge, when tri4 and tri5 are reported to be maximally expressed (Brown et al. 2011), neither GA nor ABA affected tri4, tri5, or trill gene expression
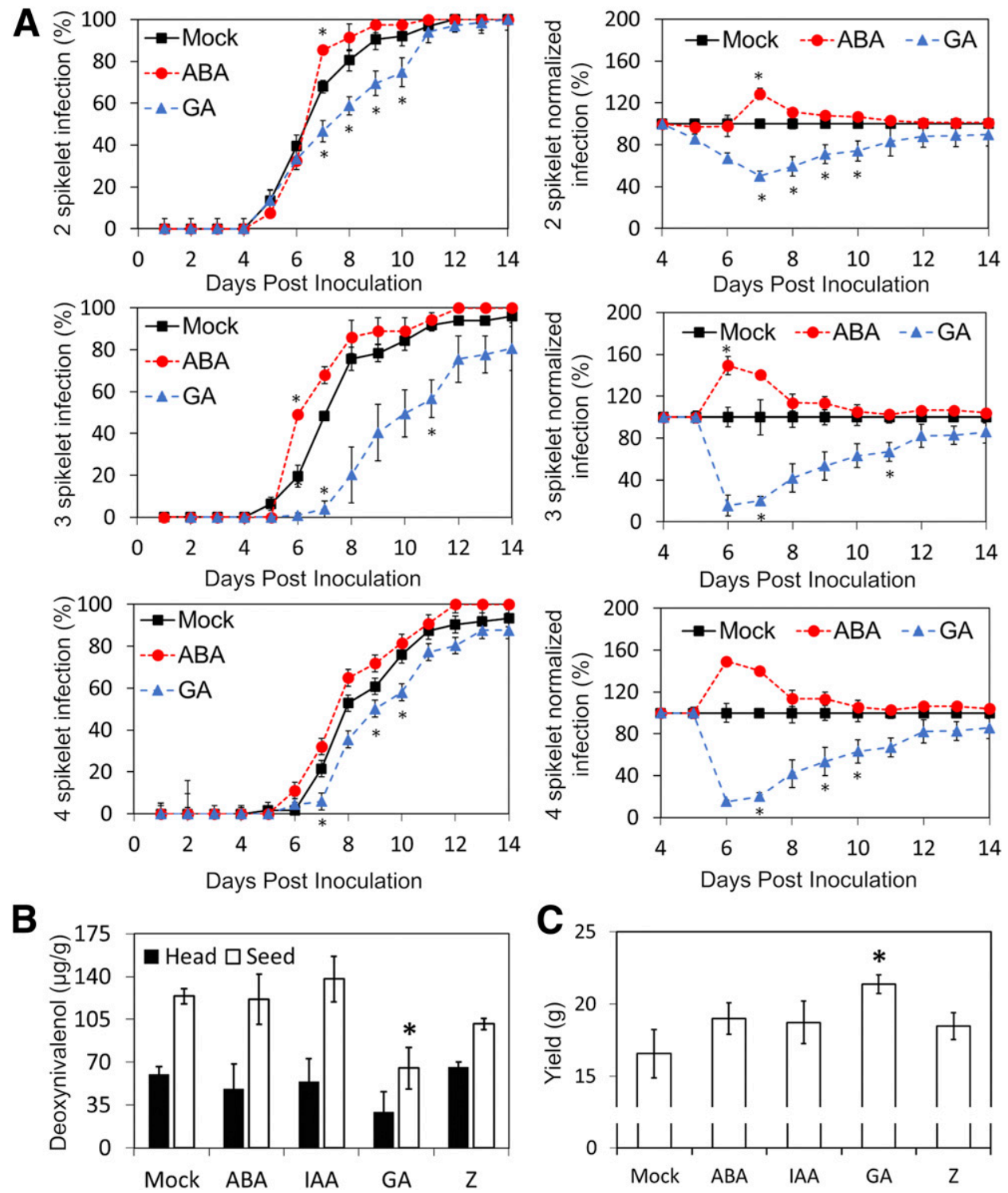

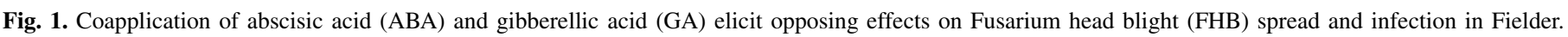

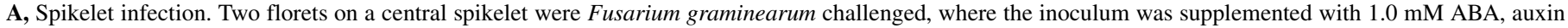

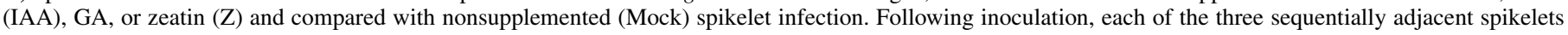

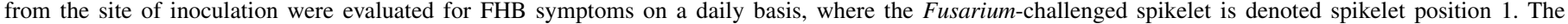

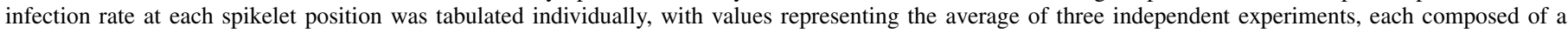

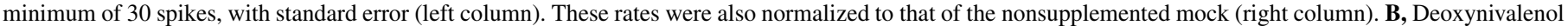

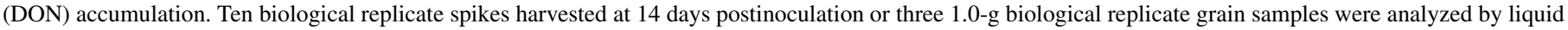

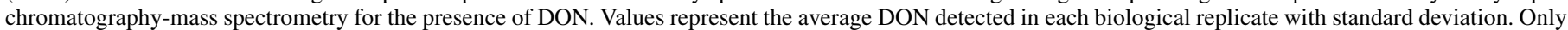

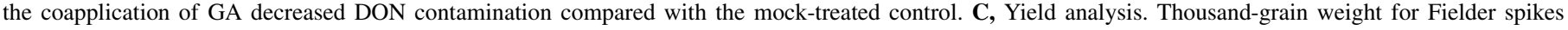

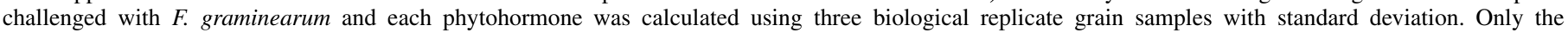

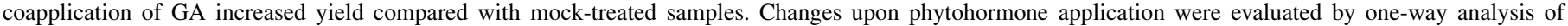
variance with Dunnett posthoc comparison (* indicates $P \leq 0.05$ ). 
relative to the actin reference gene expression (Fig. 2A). To expand on this, the effects of phytohormone treatments on expression of all of the 22 trichothecene biosynthetic genes in the pathogen were monitored in Fielder spikes by transcriptome sequencing 24 h after Fusarium challenge. Upon ABA or GA coapplication, no significant change in expression was observed $(P<0.01$ with fold change in expression $>2$ ) (Supplementary Table S1). Subsequently, the effects of $1.0 \mathrm{mM}$ ABA or GA coapplication on DON accumulation were monitored through direct toxin quantification. Fielder spikes were challenged with $F$. graminearum spores in the presence and absence of $1.0 \mathrm{mM}$ ABA or GA at each spikelet along the length of the Fielder spike, and DON was monitored 5 days postchallenge by LC-MS. Coapplication of ABA or GA did not affect the accumulation of DON in Fielder spikes (Fig. 2B).

ABA promotes the expression of hydrolases and cytoskeletal reorganization genes previously reported in early $F$. graminearum infection whereas $G A$ represses genes involved in $F$. graminearum nitrogen metabolism. Although ABA and GA do not affect $F$. graminearum tri gene expression, these phytohormones may modulate FHB infection by altering other Fusarium virulence- or infection-related gene expression. To assess this possibility, the transcriptome of $F$. graminearum was evaluated in challenged Fielder spikelets in the presence and absence of $1.0 \mathrm{mM}$ ABA or GA. As many as 140 unique $F$. graminearum genes were expressed in Fielder spikelet tissue $24 \mathrm{~h}$ after Fusarium challenge. Of these, 24 were significantly differentially expressed upon coapplication of ABA and 6 were differentially expressed upon coapplication of GA relative to that of tissue challenged with $F$. graminearum alone (Supplementary Table S2). Upon ABA coapplication, two glucosidases (FGSF_01621 and FGSF_13861), two hydrolases (FGSF_11366 and FGSF_02875), and cytoskeletal reorganization (FGSF_03563) gene expression were induced, potentially associated with promotion of plant cell wall degradation and hyphae insertion into the host wheat cells. Interestingly, when comparing genes reported to be highly expressed in diverse $F$. graminearum strains during early wheat spike infection (Lysøe et al. 2011; Menke 2011; Stephens et al. 2008), nine of these genes were observed in this work to not only be expressed but also induced two- to fivefold upon ABA coapplication (Table 2). Alternatively, upon GA coapplication, calcium-dependent aldoxime dehydratase (FGSF_06482), calcium transporting ATPase (FGSG_08985), ATP-dependent oxoprolinase (FGSF_04902), and nitroalkane oxidase (FGSF_02378) gene expression was repressed by approximately 65 to $75 \%$ while a $5^{\prime}$-nucleotidase (FGSF_00259) was induced over twofold. Because these enzymes are involved in nitrogen metabolism, regulating either inorganic nitrogenous compounds or amino acids, it is plausible that GA application hinders $F$. graminearum nitrogen-dependent bioenergetics or cellular redox (Audenaert et al. 2013). In fact, GA biosynthesis and nitrogen metabolism have been reported as opposing metabolic pathways in F. moniliforme (Mihlan et al. 2003). The remaining $F$. graminearum genes differentially expressed upon application of these hormones are poorly characterized but are putatively linked to amino acid, carbohydrate, and lipid metabolism based on DNA sequence similarity with characterized genes.

Based on the observation that ABA and GA affect $F$. graminearum early-infection gene expression, mycelium growth and sporulation
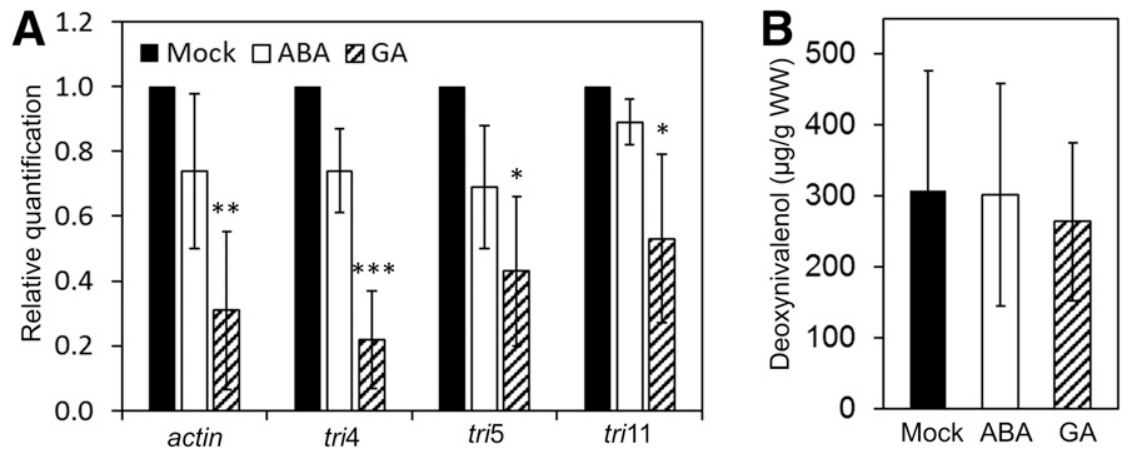

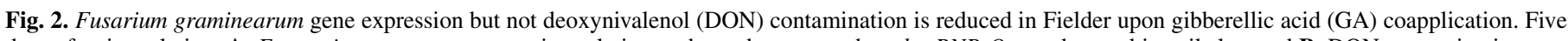

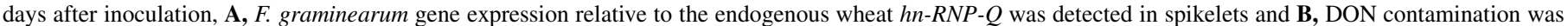

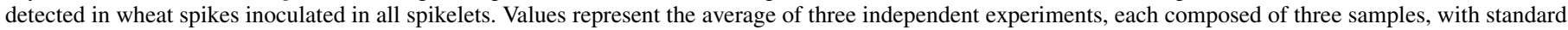

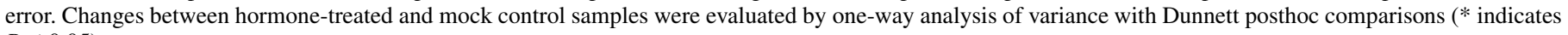
$P \leq 0.05)$.

TABLE 2. Genes involved in early Fusarium graminearum infection are induced by abscisic acid (ABA) coapplication ${ }^{\mathrm{a}}$

\begin{tabular}{|c|c|c|c|c|}
\hline Gene ID: MIPS ${ }^{\mathrm{b}}$ & Crown rot and $\mathrm{FHB}^{\mathrm{c}}$ & In vivo infection ${ }^{\mathrm{d}}$ & Global FHB & ABA induced \\
\hline FGSG_03632: Related to cellulose binding CEL1 & $\sqrt{ }$ & $\sqrt{ }$ & $\sqrt{ }$ & $\sqrt{ }$ \\
\hline FGSG_07642: Related to monooxigenase & $\ldots$ & $\sqrt{ }$ & $\sqrt{ }$ & $\sqrt{ }$ \\
\hline FGSG_10989: conserved hypothetical protein & $\ldots$ & $\ldots$ & $\sqrt{ }$ & $\sqrt{ }$ \\
\hline $\begin{array}{l}\text { FGSG_10991: Related to benzoate 4-monooxygenase } \\
\text { cytochrome P450 }\end{array}$ & $\cdots$ & $\sqrt{ }$ & $\sqrt{ }$ & $\sqrt{ }$ \\
\hline FGSG_10992: Related to polysaccharide deacetylase & $\ldots$ & $\sqrt{ }$ & $\sqrt{ }$ & $\sqrt{ }$ \\
\hline FGSG_10993: Related to selenocysteine lyase & $\ldots$ & $\sqrt{ }$ & $\cdots$ & $\sqrt{ }$ \\
\hline FGSG_11366: Conserved hypothetical protein & $\ldots$ & $\sqrt{ }$ & $\cdots$ & $\sqrt{ }$ \\
\hline FGSG_11397: Related to desaturase & $\ldots$ & $\ldots$ & $\sqrt{ }$ & $\sqrt{ }$ \\
\hline FGSG_11399: Related to oxidoreductase & $\ldots$ & $\sqrt{ }$ & $\sqrt{ }$ & $\sqrt{ }$ \\
\hline
\end{tabular}

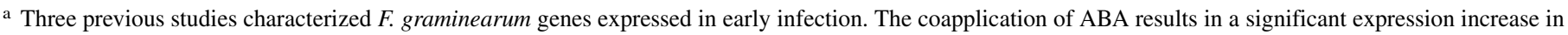
at least nine of these genes.

b F. graminearum gene ID and Munich Information Center for Protein Sequences (MIPS) annotation.

c Fusarium head blight (FHB) (Stephens et al. 2008).

d Menke 2011.

e Lysøe et al. 2011. 
were monitored to determine whether the ABA- and GA-mediated modulation of FHB spread (Fig. 1) is the direct effect of these compounds on $F$. graminearum viability, as opposed to phytohormoneinduced host resistance. PBZ, a triazole fungicide that inhibits ergosterol biosynthesis in Fusarium fungal species (Vanden Bossche et al. 1989), was used as a positive control for fungicidal activity. The presence of $1.0 \mathrm{mM}$ ABA, IAA, GA, or Z did not affect spore germination or mycelium production on rich media from isolated spores or fungal plugs (Fig. 3A and B). Furthermore, these phytohormones also did not affect $F$. graminearum growth in rich liquid media (Fig. 3C) or production of spores in the presence of cellulose (Fig. 3D). However, ABA, GA, and $\mathrm{Z}$ did alter the fungal morphology of $F$. graminearum in liquid media where the formation of condensed fungal masses, rather than diffuse mycelia, was observed (Fig. 3E).

Opposing effects of ABA and GA on FHB spread are not related to modification of the classical SA or JA defense phytohormone pathways in Fielder spikes. Due to extensive hormonal cross-talk among phytohormone signaling pathways during fungal disease responses (De Vleesschauwer et al. 2014), the effects of hormone application on FHB pathogenesis may elicit synergistic or antagonistic interactions with additional classical defense responsive phytohormones. To test the effects on JA and SA phytohormone content, Fielder spikes were challenged with $F$. graminearum spores in the presence and absence of $1.0 \mathrm{mM} \mathrm{ABA}$ or GA by point inoculation at two central spikelets. The phytohormone content from the treated individual Fielder spikes was determined 7 days after Fusarium challenge, when the differential effects of ABA and GA on disease response are most obvious (Fig. 1). The phytohormone profile of
Fusarium-challenged spikes revealed an increase in JA accumulation as compared with unchallenged spikes, consistent with a previous report (Buhrow et al. 2016), whereas the coapplication of ABA or GA with $F$. graminearum did not alter JA or SA phytohormone content compared with pathogen challenge alone (Table 3).

Phytohormone applications may serve as a stand-alone or integrative approach to mitigating FHB in susceptible wheat cultivars. Based on the observation that $\mathrm{ABA}$ and GA differentially modulate FHB spread in Fielder, one strategy to address the agronomic and economic costs of $F$. graminearum wheat infection is the development of potent, stable phytohormone analogs. However, the utility of phytohormone and phytohormone analog applications is dependent on the effect of these molecules on grain yield and quality. To investigate the effects of hormone treatments on grain production and viability independently of FHB infection, 1.0 mM IAA, ABA, GA, Z, or solvent control were applied to Fielder spikes at each spikelet during anthesis. There were no visible phenotypic differences in mature grain harvested from phytohormones-treated spikes compared with those from control spikes (Fig. 4A). Although ABA, IAA, and GA led to a slight but significant reduction, $\mathrm{Z}$ led to a slight but significant increase in yield (Fig. 4B). Seed germination rates were increased by both GA and $\mathrm{Z}$ spike treatments and decreased by ABA spike treatment as compared with solvent-treated controls (Fig. 4C). These results suggest that single applications of ABA, GA, or related chemical analogs during anthesis have only minor effects on development of wheat grains. Thus, their application may be a viable approach for the modulation of FHB severity, which has a much more significant impact on grain yield and quality.
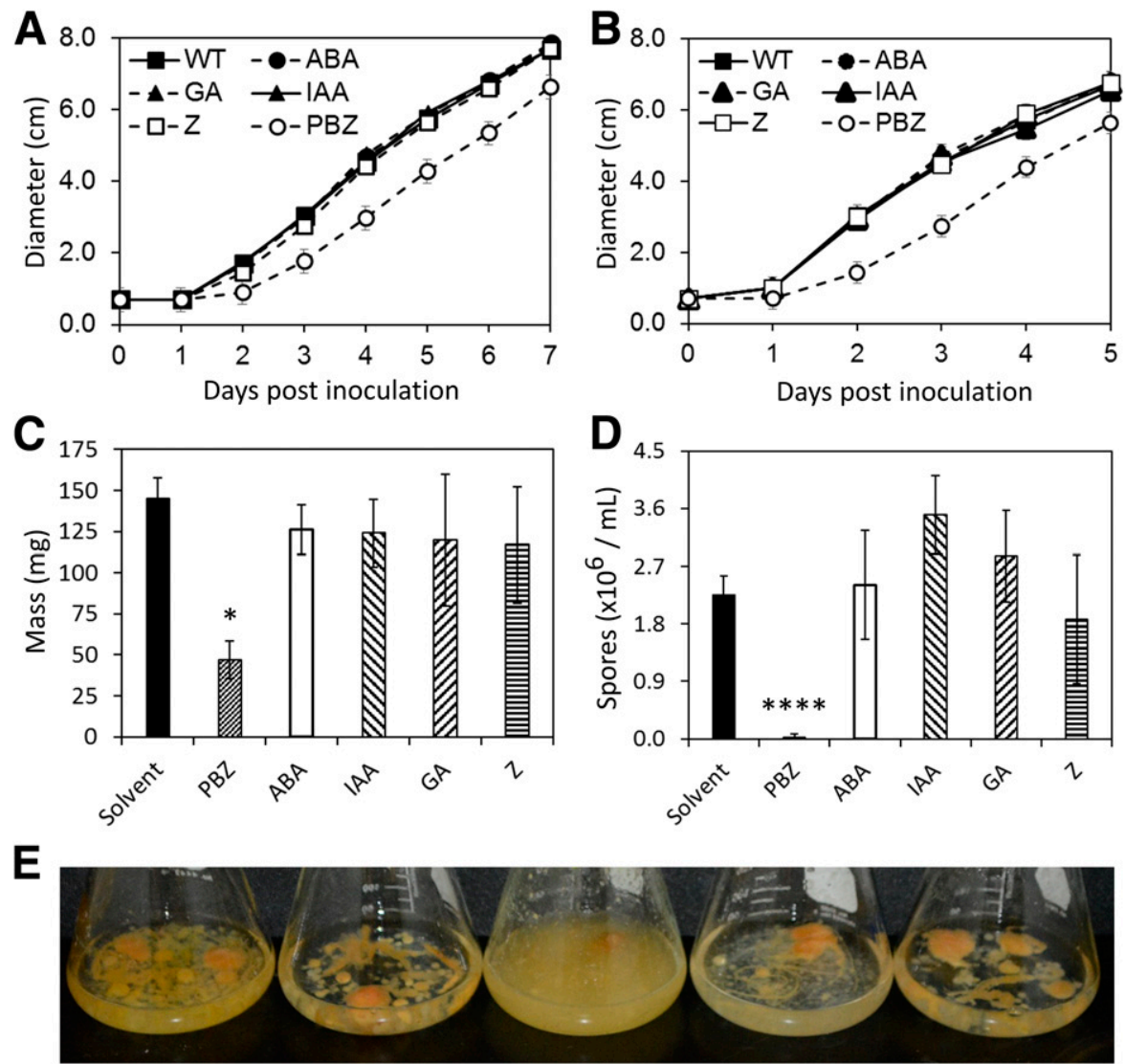

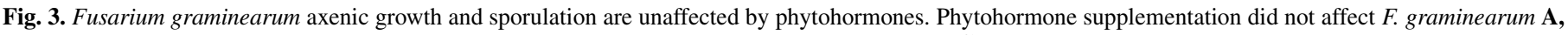

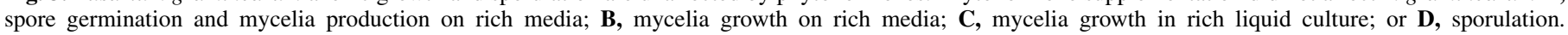

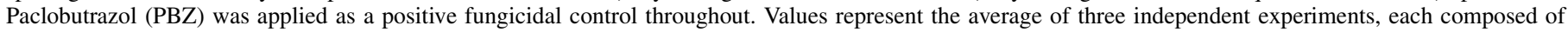

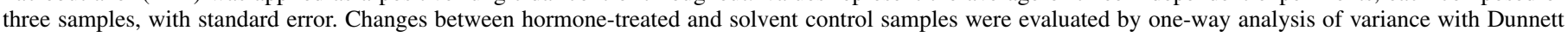

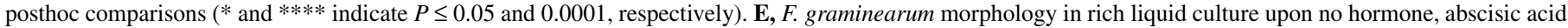
(ABA), auxin (IAA), gibberellic acid (GA), or zeatin (Z) (left to right) supplementation. 
An ABA antagonist, 3'-hexasulfanyl-(+)-ABA (AS6), has recently been synthesized and characterized (Takeuchi et al. 2014). AS6 competitively binds to Arabidopsis thaliana ABA receptors and, through its hexysulfanyl group, sterically hinders the formation of ABA receptor protein phosphatase $2 \mathrm{C}$ complexes and subsequent signaling (Takeuchi et al. 2014). To test whether coapplication of AS6 is capable of reducing FHB phenotypic spread, Fielder spikes were challenged with $F$. graminearum supplemented with $1.0 \mathrm{mM}$ AS6. AS6 did not significantly alter FHB spread, DON contamination, or yield (Supplementary Fig. S4). The lack of AS6 efficacy on FHB spread may be a result of reduced chemical stability, bioavailability, metabolic half-life, or wheat receptor affinity compared with ABA.

An alternative strategy to address the costs of $F$. graminearum infection is the combined application of novel phytohormone modulators and existing Fusarium fungicides such that multiple metabolic pathways are targeted. GA may inhibit FHB spread by disrupting Fusarium spp. nitrogen acquisition and metabolism, while PBZ has been shown to target ergosterol-rich fungal cell membranes (Vanden Bossche et al. 1989). To investigate whether GA and PBZ can be combined to more effectively reduce Fusarium pathogenesis, Fielder spikes were $F$. graminearum challenged at a central spikelet, where the inoculum was supplemented with $1.0 \mathrm{mM}$ GA, $10 \mu \mathrm{M}$ PBZ, or the combination of GA and PBZ. To enable this experiment, it was necessary to dissolve PBZ and, thus,

TABLE 3. Abscisic acid (ABA) and gibberellic acid (GA) coapplication do not affect salicylic acid (SA) or jasmonic acid (JA) hormone profiles in Fusariumchallenged Fielder spikes ${ }^{\mathrm{a}}$

\begin{tabular}{lcccc}
\hline & & \multicolumn{3}{c}{ Fusarium challenged } \\
\cline { 3 - 5 } Phytohormones (ng/g) & Mock & Mock & ABA treated & GA treated \\
\hline SA & $56 \pm 11$ & $71 \pm 25$ & $66 \pm 11$ & $53 \pm 11$ \\
Conjugated SA & $667 \pm 87$ & $809 \pm 333$ & $528 \pm 127$ & $478 \pm 102$ \\
JA & $21 \pm 3$ & $39 \pm 12^{*}$ & $65 \pm 34$ & $55 \pm 10$ \\
JA-Ile & $21 \pm 5$ & $23 \pm 7$ & $21 \pm 8$ & $21 \pm 2$ \\
\hline
\end{tabular}

a Values represent the average phytohormone content of five biological Fielder spike replicates with standard deviation at 7 days post Fusarium challenge or after mock inoculation. Phytohormone differences between ABA or GA coapplication and the mock control were evaluated by one-way analysis of variance with Dunnett posthoc comparisons. A significant increase in JA content (* indicates $P<0.05$ ) was observed upon Fusarium graminearum challenging compared with mock (deionized water) treatment. Ile $=$ isoleucine.
GA as well in $10 \%(\mathrm{vol} / \mathrm{vol})$ methanol rather than the initially selected deionized water solvent. The application of alternative solvent systems may affect the rate of FHB spread in the absence of fungicide or phytohormone coapplication; however, any apparent differences in spread using water (Fig. 1) or 10\% (vol/vol) methanol solvents (Fig. 5) were not statistically significant in this work. Coapplication of $1.0 \mathrm{mM}$ GA or $10 \mu \mathrm{M}$ PBZ alone moderately reduced FHB spread in spikelets beginning 7 days postinoculation, while the coapplication of $1.0 \mathrm{mM} \mathrm{GA}$ and $10 \mu \mathrm{M}$ PBZ further reduced FHB phenotypic symptoms in $F$. graminearum-challenged Fielder spikes (Fig. 5). The additive effects of GA and PBZ are recapitulated by reduced DON contamination in Fielder spikes and grain and increased yield (Fig. 5B and C).

\section{DISCUSSION}

FHB is a costly disease affecting wheat and other small-grain cereal development and commercial viability. Consistent with previous observations, phytohormone profiling in this work describes the induction of SA and JA metabolism but, additionally, implicates ABA biosynthesis and metabolism upon $F$. graminearum challenge in both FHB-susceptible and -resistant cultivars. Unique to the FHB-resistant Sumai 3, cytokinin and GA metabolism is promoted in unchallenged spike tissue, suggesting that phytohormones not traditionally reported to be involved in biotic stress may provide underlying physiological characteristics related to inherent resistance. This possibility may be further explored by comparing hormone profiles of multiple wheat varieties with varying degrees of FHB susceptibility.

In addition to the classical defense response phytohormones, the application of $\mathrm{ABA}$ and $\mathrm{GA}$ were also shown to modulate pathogenesis in wheat, with GA promoting resistance and ABA promoting susceptibility. During the preparation of this work, Qi et al. (2016) reported that multiple applications of millimolar concentrations of racemic $\mathrm{ABA}$ solutions, rather than the single application of (+)-ABA in this work, with and after $F$. graminearum challenge are capable of increasing FHB symptoms in susceptible 'Roblin' wheat. In both reports, these effects were observed most strongly in the early stages of FHB spread from challenged tissue to an adjacent asymptomatic spikelet. This specific response suggests that the ABA-elicited effects are limited to early infection spread or have limited duration or localization. Together, these findings strengthen the idea that ABA plays a role in promoting FHB and

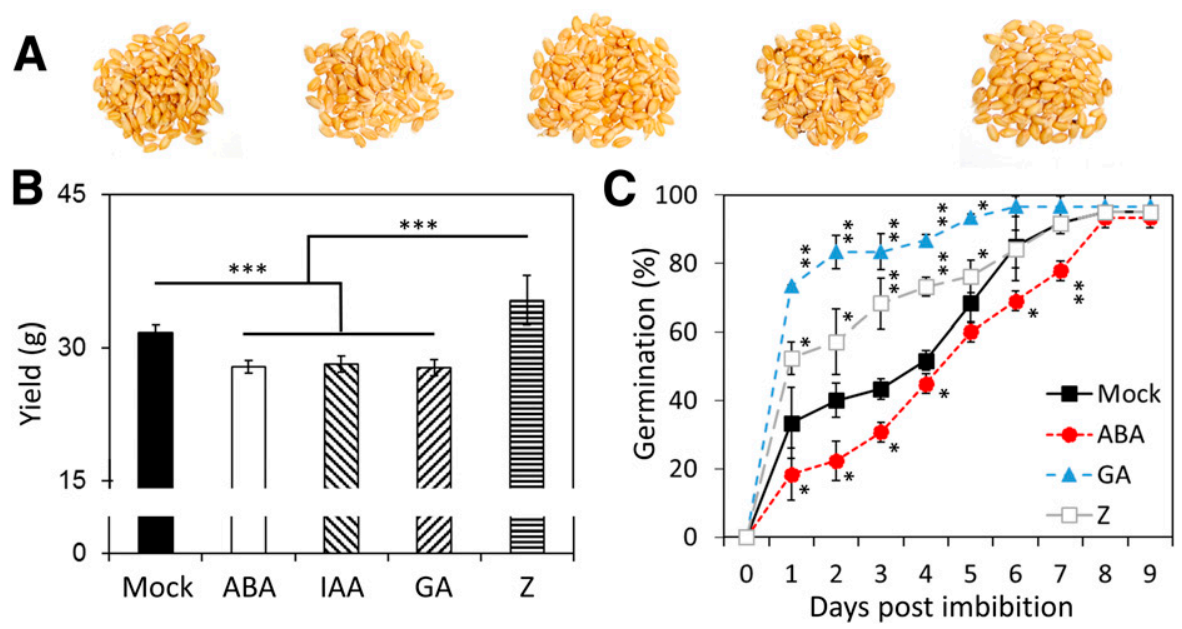

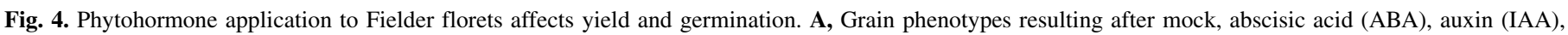

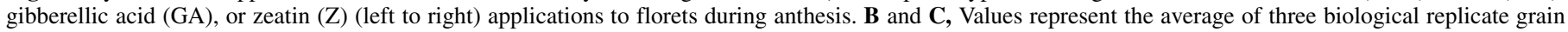

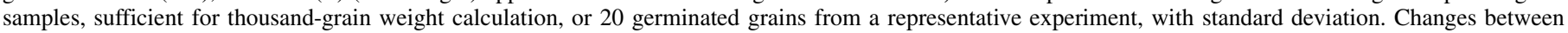

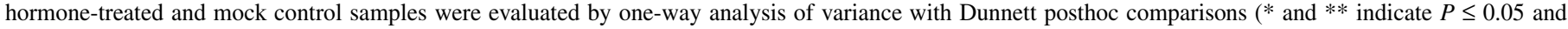
0.001 , respectively). 
further suggest that an $\mathrm{ABA}$ antagonist, similar to the recently reported AS6, may be exogenously applied to reduce FHB spread in diverse susceptible wheat lines.

The mechanism of ABA and GA modulation of FHB disease progression was evaluated in terms of a direct effect on the $F$. graminearum fungus and also with respect to previously reported $F$. graminearum-elicited wheat phytohormone responses. The coapplication of ABA promotes $F$. graminearum early-stage gene expression, potentially promoting wheat pathogenesis by cell wall degradation and fungal insertion into challenged spikes. Additionally, the coapplication of GA represses early-stage $F$. graminearum nitrogen metabolic gene expression, potentially reducing bioenergetic resources and redox regulation required for the newly infecting fungal cells. Although ABA and GA elicit Fusarium gene expression changes that would be expected to promote and repress FHB pathogenesis, respectively, these phytohormones may also elicit later-stage regulation of fungal expression events or act on the wheat host. The application of ABA and GA did not have a significant effect on $F$. graminearum growth or sporulation in axenic conditions. Reports of ABA on $F$. graminearum axenic growth are mixed; in rich media, no growth defects are observed (this work) (Petti et al. 2012), whereas high micromolar concentrations of ABA inhibited mycelia growth but not spore germination in minimal media (Qi et al. 2016). Finally, ABA and GA application did not alter trichothecene gene cluster expression, DON accumulation, or SA or JA biosynthesis in $F$. graminearum-challenged spikes.

Although the application of (+)-ABA and GA (predominantly GA3) affect FHB spread in Fielder spikes, the identities of the bioactive molecules responsible for eliciting these effects remain
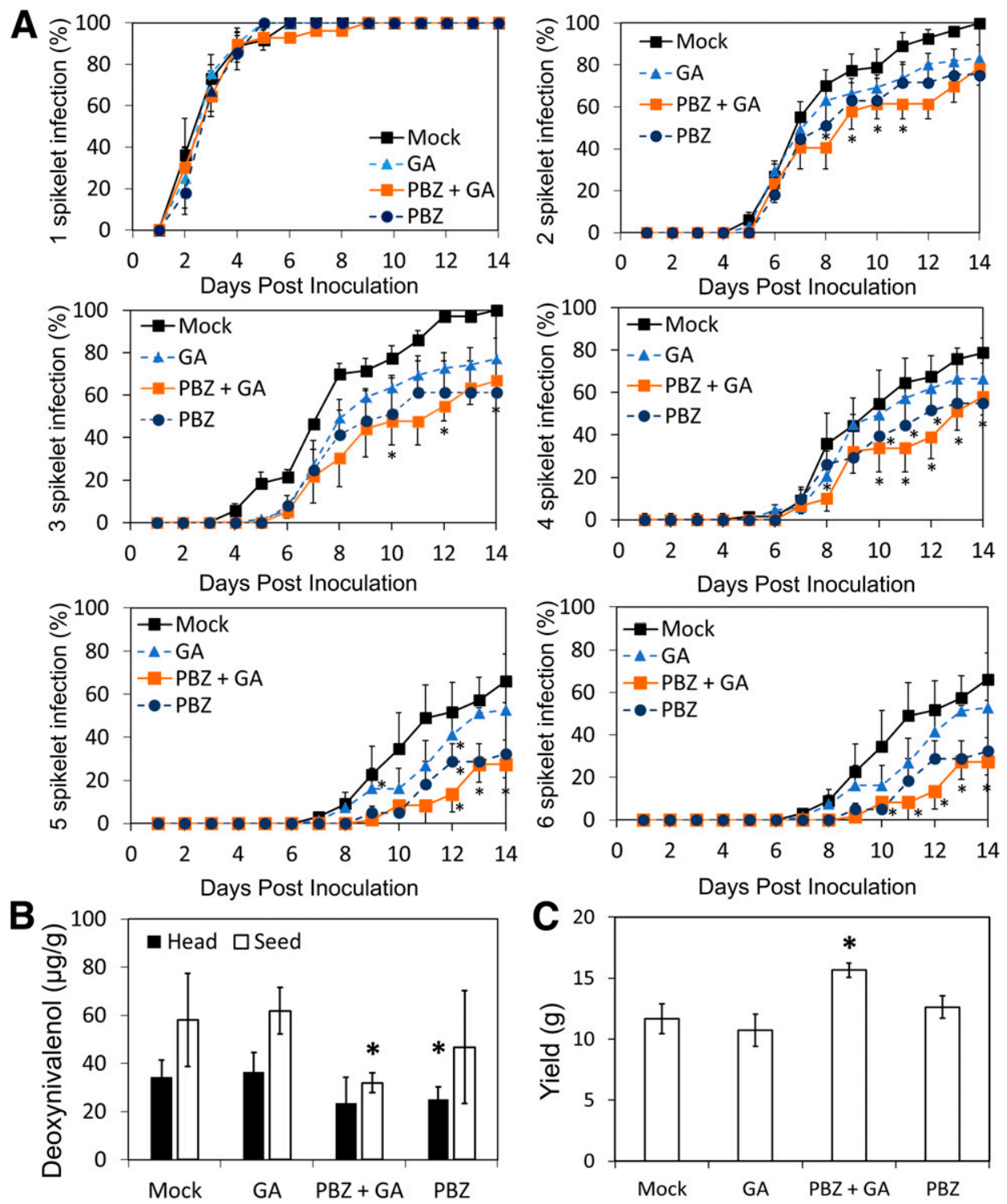

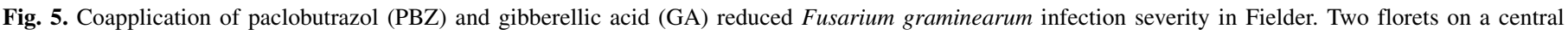

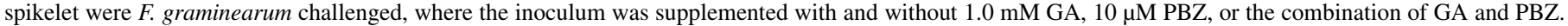

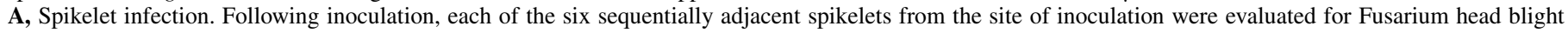

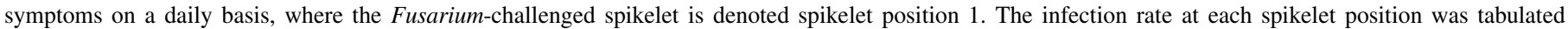

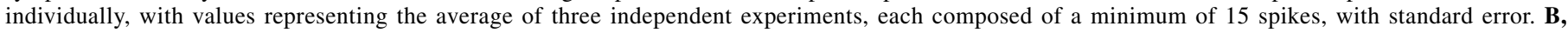

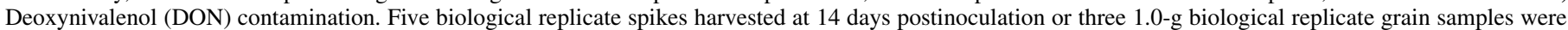

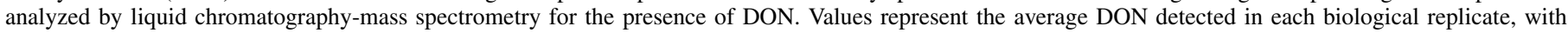

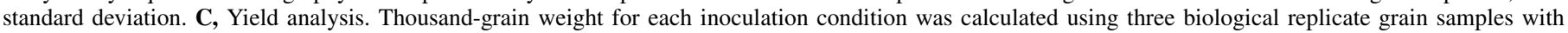

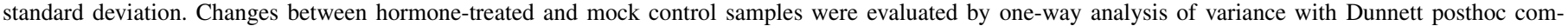
parisons (* indicates $P \leq 0.05$ ). 
unknown. ABA is a sesquiterpene phytohormone, synthesized in plants by the carotenoid-dependent, plastidal nonmevalonate biosynthetic pathway (Nambara and Marion-Poll 2005). Additionally, a number of plant-symbiotic and -pathogenic fungal species have also been shown to biosynthesize ABA (Crocoll et al. 1991; Dörffling et al. 1984; Siewers et al. 2006), with an F. graminearum strain reported to biosynthesize low-nanogram concentrations of ABA in axenic culture (Qi et al. 2016). Alternatively, GA is a diterpenoid phytohormone that is biosynthesized through the methylerythritol phosphate pathway, where more than 20 GA have been identified (Yamaguchi 2008). A number of fungal and bacterial species are also capable of synthesizing GA (MacMillan 2001), including mating populations of $F$. fujikuroi (Yabuta 1935). Furthermore, GA has been reported to be moderately unstable in aqueous solution (Pryce 1973a,b); therefore, the use of alternative solvent systems, such as the $10 \%$ methanol applied in this work to investigate the combined contribution of GA and PBZ, may alter the chemical stability and metabolism of exogenously applied GA. Together, these reports highlight the possibility that the bioactive molecules responsible for eliciting changes on FHB spread may be wheator $F$. graminearum-derived phytohormone metabolites or their chemical degradative products. Toward elucidating the metabolic fate and duration of exogenous and endogenous phytohormones, the hormone profiles arising from coapplication of labeled phytohormones to $F$. graminearum-challenged Fielder spikes are being evaluated.

In summary, the phytohormone profiling documented in this work supports the biosynthesis of SA, JA, and $\mathrm{ABA}$ in response to $F$. graminearum challenge, and the idea that this effect is independent of the degree of FHB susceptibility of the wheat cultivar. ABA and GA were shown to modulate FHB spread in an opposing manner when coapplied to susceptible Fielder spikes, where ABA was shown to promote $F$. graminearum gene expression linked to early wheat infection and GA was shown to repress fungal nitrogen metabolism; however, a full understanding of the associated mechanisms remains to be determined. Finally, based on the combined effect of GA and PBZ on limiting FHB spread, this work supports an agronomic strategy of combining phytohormonerelated treatments with existing fungicides and biocontrol agents for the management of FHB in wheat. Interestingly, PBZ itself may reduce FHB disease progress not only by acting as a fungicide but also by modulating wheat phytohormone biosynthesis and signaling because this molecule has been reported to inhibit GA (Brock et al. 2011) and ABA (Norman et al. 1986) biosynthesis in fungal plant pathogens and SA biosynthesis in planta (Leon et al. 1995). Overall, this study implicates the role of diverse phytohormones in regulating FHB pathogenesis in wheat, potentially by eliciting wheat responses and acting on the $F$. graminearum transcriptome.

\section{ACKNOWLEDGMENTS}

We thank L. I. Zaharia at the Hormone Profiling Service, National Research Council of Canada, Saskatoon, Canada, for data collection and methodological input; S. McCormick at the United State Department of Agriculture for providing the $F$. graminearum strain GZ3639; K. Nelson and S. Abrams at the University of Saskatchewan for providing AS6; and E. Liu at the National Research Council of Canada for providing Fusarium actin-specific primers. Funding for this work to M. C. Loewen, N. A. Foroud, and D. Tulpan was provided by the National Research Council of Canada-Wheat Flagship, as part of the Canadian Wheat Alliance. This manuscript represents National Research Council of Canada Communication number 56135.

\section{LITERATURE CITED}

Abrams, S. R., Nelson, K., and Ambrose, S. J. 2003. Deuterated abscisic acid analogs for mass spectrometry and metabolism studies. J. Labelled Compounds Radiopharm. 46:273-283.
Ali, S. S., Kumar, G. B., and Doohan, F. M. 2013. Brassinosteroid enhances resistance to Fusarium diseases of barley. Phytopathology 103:1260-1267.

Ameye, M., Audenaert, K., De Zutter, N., Steppe, K., Van Meulebroek, L., Vanhaecke, L., De Vleesschauwer, D., Haesaert, G., and Smagghe, G. 2015. Priming of wheat with the green leaf volatile Z-3-hexenyl acetate enhances defense against Fusarium graminearum but boosts deoxynivalenol production. Plant Physiol. 167:1671-1684.

Anders, S., Pyl, P. T., and Huber, W. 2015. HTSeq-A Python framework to work with high-throughput sequencing data. Bioinformatics 31:166-169.

Antonissen, G., Martel, A., Pasmans, F., Ducatelle, R., Verbrugghe, E., Vandenbroucke, V., Li, S., Haesebrouck, F., Van Immerseel, F., and Croubels, S. 2014. The impact of Fusarium mycotoxins on human and animal host susceptibility to infectious diseases. Toxins (Basel) 6:430-452.

Audenaert, K., Vanheule, A., Hofte, M., and Haesaert, G. 2013. Deoxynivalenol: A major player in the multifaceted response of Fusarium to its environment. Toxins (Basel) 6:1-19.

Bai, G. H., and Shaner, G. E. 2004. Management and resistance in wheat and barley to Fusarium head blight. Annu. Rev. Phytopathol. 42:135-161.

Bolger, A. M., Lohse, M., and Usade, B. 2014. Trimmomatic: A flexible trimmer for Illumina Sequence Data. Bioinformatics 30:2114-2120.

Brock, N. L., Tudzynski, B., and Dickschat, J. S. 2011. Biosynthesis of sesqui- and diterpenes by the gibberellin producer Fusarium fujikuroi. ChemBioChem 12:2667-76.

Brown, N. A., Bass, C., Baldwin, T. K., Chen, H., Fabien, M., Carion, P. W. C., Urban, M., van de Meene, A. M. L., and Hammond-Kosack, K. E. 2011. Characterization of Fusarium graminearum-wheat floral interaction. J. Pathogens 2011: Article ID 626345. doi:10.4061/2011/626345

Buhrow, L. M., Clark, S. M., and Loewen, M. C. 2016. Identification of an attenuated Barley Stripe Mosaic Virus for the virus-induced gene silencing of pathogenesis-related wheat genes. Plant Methods 12:12.

Casacuberta, J. M., Raventós, D., Puigdoménech, P., and San Segundo, B. 1992. Expression of the gene encoding the PR-like protein PRms in germinating maize embryos. Mol. Gen. Genet. 234:97-104.

Chen, X., Steed, A., Travella, S., Keller, B., and Nicholson, P. 2009. Fusarium graminearum exploits ethylene signalling to colonize dicotyledonous and monocotyledonous plants. New Phytol. 182:975-983.

Crocoll, C., Kettner, J., and Dörffling, K. 1991. Abscisic acid in saprophytic and parasitic species of fungi. Phytochemistry 30:1059-1060.

Cuomo, C. A., Güldener, U., Xu, J.-R., Trail, F., Turgeon, B. G., Di Pietro, A., Walton, J. D., Ma, L.-J., Baker, S. E., Rep, M., Adam, G., Antoniw, J., Baldwin, T., Calvo, S., Chang, Y.-L., Decaprio, D., Gale, L. R., Gnerre, S., Goswami, R. S., Hammond-Kosack, K., Harris, L. J., Hilburn, K., Kennell, J. C., Kroken, S., Magnuson, J. K., Mannhaupt, G., Mauceli, E., Mewes, H.-W., Mitterbauer, R., and Muehlbauer, G. 2007. The Fusarium graminearum genome reveals a link between localized polymorphism and pathogen specialization. Science 317:1400-1402.

De Vleesschauwer, D., Jing, X., and Hofte, M. 2014. Making sense of hormone-mediated defense networking: From rice to Arabidopsis. Front. Plant Sci. 5:00611.

Diethelm, M., Schmolke, M., Groth, J., Friedt, W., Schweizer, G., and Hartl, L. 2014. Association of allelic variation in two NPR1-like genes with Fusarium head blight resistance in wheat. Mol. Breed. 34:31-43.

Ding, L., Xu, H., Yi, H., Yang, L., Kong, Z., Zhang, L., Xue, S., Jia, H., and Ma, Z. 2011. Resistance to hemi-biotrophic F. graminearum infection is associated with coordinated and ordered expression of diverse defense signaling pathways. PLoS One 6:e19008.

Divon, H. H., Ziv, C., Davydov, O., Yarden, O., and Fluhr, R. 2006. The global nitrogen regulator, FNR1, regulates fungal nutrition-genes and fitness during Fusarium oxysporum pathogenesis. Mol. Plant Pathol. 7: 485-497.

Dobin, A., Davis, C. A., Schlesinger, F., Drenkow, J., Zaleski, C., Jha, S., Batut, P., Chaisson, M., and Gingeras, T. R. 2012. STAR: Ultrafast universal RNA-seq aligner. Bioinformatics 29:15-21.

Dörffling, K., Petersen, W., Sprecher, E., Urbasch, I., and Hanssen, H. P. 1984. Abscisic acid in phytopathogenic fungi of the genera Botrytis, Ceratocystis, Fusarium, and Rhizoctonia. Z. Naturforsch. C 39:683-684.

Edwards, S. G. 2004. Influence of agricultural practices on Fusarium infection of cereals and subsequent contamination of grain by trichothecene mycotoxins. Toxicol. Lett. 153:29-35.

Eshel, D., Lichter, A., Dinoor, A., and Prusky, D. 2002. Characterization of Alternaria alternata glucanase genes expressed during infection of resistant and susceptible persimmon fruits. Mol. Plant Pathol. 3:347-358.

Gale, L. R. 2003. Population biology of Fusarium species causing head blight of grain crops. Pages 120-143 in: Fusarium Head Blight of Wheat and Barley. K. J. Leonard and W. R. Bushnell, eds. American Phytopathological Society, St. Paul, MN.

Galka, P. W. S., Ambrose, S. J., Ross, A. R. S., and Abrams, A. R. 2005. Synthesis of deuterated jasmonates for mass spectrometry and metabolism studies. J. Labelled Compounds Radiopharm. 48:797-809. 
Kang, Z., and Buchenauer, H. 1999. Immunocytochemical localization of Fusarium toxins in infected wheat spikes by Fusarium culmorum. Physiol. Mol. Plant Pathol. 55:275-288.

Kang, Z., and Buchenauer, H. 2000. Ultrastructural and immunochtychemical investigation of pathogen development and host responses in resistant and susceptible wheat spikes infected with Fusarium culmorum. Physiol. Mol. Plant Pathol. 57:255-268.

Kulik, T., Lojko, M., Jestoi, M., and Perkowski, J. 2012. Sublethal concentrations of azoles induce tri transcript levels and trichothecene production in Fusarium graminearum. FEMS Microbiol. Lett. 335:58-67.

Leon, J., Lawton, M. A., and Raskin, L. 1995. Hydrogen peroxide stimulates salicylic acid biosynthesis in tobacco. Plant Physiol. 108:1637-1678.

Li, G., and Yen, Y. 2008. Jasmonate and ethylene signaling pathway may mediate Fusarium head blight resistance in wheat. Crop Sci. 48:1888-1896.

Livak, K. J., and Schmittgen, T. D. 2001. Analysis of relative gene expression data using real- time quantitative PCR and the $2^{-\triangle \Delta C T}$ method. Methods 25 : 402-408.

Love, M. I., Huber, W., and Anders, S. 2014. Moderated estimation of fold change and dispersion for RNA-seq data with DESeq2. Genome Biol. 15:550.

Lulsdorf, M. M., Yuan, H. Y., Slater, S. M. H., Vandenberg, A., Han, X., Zaharia, L. I., and Abrams, S. R. 2013. Endogenous hormone profiles during early seed development of $C$. arietinum and $C$. anatolicum. J. Plant Growth Regul. 71:191-198.

Lysøe, E., Seong, K.-Y., and Kistler, H. C. 2011. The transcriptome of Fusarium graminearum during the infection of wheat. Mol. Plant-Microbe Interact. 24:995-1000.

MacMillan, J. 2001. Occurrence of gibberellins in vascular plants, fungi, and bacteria. J. Plant Growth Regul. 20:387-442.

Makandar, R., Essig, J. S., Schapaugh, M. A., Trick, H. N., and Shah, J. 2006. Genetically engineered resistance to Fusarium head blight in wheat by expression of Arabidopsis NPR1. Mol. Plant-Microbe Interact. 19:123-129.

Makandar, R., Nalam, V. J., Chaturvedi, R., Jeannotte, R., Sparks, A. A., and Shah, J. 2010. Involvement of salicylate and jasmonate signaling pathways in Arabidopsis interaction with Fusarium graminearum. Mol. PlantMicrobe Interact. 23:861-870.

Makandar, R., Nalam, V. J., Lee, H., Trick, H. N., Dong, Y., and Shah, J. 2012. Salicylic acid regulates basal resistance to Fusarium head blight in wheat. Mol. Plant-Microbe Interact. 25:431-439.

McCormick, S. 2003. The role of DON in pathogenicity. Pages 165-183 in: Fusarium Head Blight of Wheat and Barley. K. J. Leonard and W. R. Bushnell, eds. American Phytopathological Society, St. Paul, MN.

McMullen, M., Jones, R., and Gallenberg, D. 1997. Scab of wheat and barley: A re-emerging disease of devastating impact. Plant Dis. 81:1340-1348.

Menke, J. R. 2011. A study of Fusarium graminearum virulence factors. Ph.D. dissertation, University of Minnesota, St. Paul.

Mesterházy, Á., Bartok, T., and Lamper, C. 2003. Influence of wheat cultivar, species of Fusarium, and isolate aggressiveness on the efficacy of fungicides for control of Fusarium head blight. Plant Dis. 87:1107-1115.

Mihlan, M., Homann, V., Liu, T. W., and Tudzynski, B. 2003. AREA directly mediates nitrogen regulation of gibberellin biosynthesis in Gibberella fujikuroi, but its activity is not affected by NMR. Mol. Microbiol. 47:975-991.

Mostrom, M. S., and Raisbeck, M. F. 2007. Trichothecenes. Pages 951-976 in: Veterinary Toxicology, 1st ed. R. C. Gupta, ed. Elsevier, New York.

Nambara, E., and Marion-Poll, A. 2005. Abscisic acid biosynthesis and catabolism. Annu. Rev. Plant Biol. 56:165-185.

Namiki, F., Matsunaga, M., Okuda, M., Inoue, I., Nishi, K., Fujita, Y., and Tsuge, T. 2001. Mutation of an arginine biosynthesis gene causes reduced pathogenicity in Fusarium oxysporum f. sp. melonis. Mol. Plant-Microbe Interact. 14:580-584.

Norman, S. M., Bennett, R. D., Poling, S. M., Maier, V. P., and Nelson, M. D. 1986. Paclobutrazol inhibits abscisic acid biosynthesis in Cercospora rosicola. Plant Physiol. 80:122-125.

Parry, D. W., Jenkinson, P., and McLeod, L. 1995. Fusarium ear blight (scab) in small grain cereals-A review. Plant Pathol. 44:207-238.

Pask, A. J. D., Pietragalla, J., Mullan, D. M., and Reynolds, M. P. 2012. Physiological Breeding II: A Field Guide to Wheat Phenotyping, IV. CIMMYT, Mexico, DF.

Petti, C., Reiber, K., Ali, S. S., Berney, M., and Doohan, F. M. 2012. Auxin as a player in the biocontrol of Fusarium head blight disease of barley and its potential as a disease control agent. BMC Plant Biol. 12:224.
Plattner, D. J., and Maragos, C. M. 2003. Determination of deoxynivalenol and nivalenol in corn and wheat by liquid chromatography with electrospray mass spectrometry. J. AOAC Int. 86:61-65.

Proctor, R. H., Hohn, T. M., and McCormick, S. P. 1995. Reduced virulence of Gibberella zeae caused by disruption of a trichothecene toxin biosynthesis gene. Mol. Plant-Microbe Interact. 8:593-601.

Pryce, R. J. 1973a. Decomposition of aqueous solutions of gibberellic acid on autoclaving. Phytochemistry 12:507-514.

Pryce, R. J. 1973b. Allogibberic acid: An inhibitor of flowering in Lemna perpusilla. Phytochemistry 12:1745-1754.

Qi, P.-F., Balcerzak, M., Rocheleau, H., Leung, W., Wei, Y.-M., Zheng, Y.-L., and Ouellet, T. 2016. Jasmonic acid and abscisic acid play important roles in host-pathogen interaction between Fusarium graminearum and wheat during the early infection stages of Fusarium head blight. Physiol. Mol. Plant Pathol. 93:39-48.

Qi, P.-F., Johnson, A., Balcerzak, M., Rocheleau, H., Harris, L. J., Long, X. Y., Wei, Y. M., Zheng, Y. L., and Ouellet, T. 2012. Effect of salicylic acid on Fusarium graminearum, the major causal agent of Fusarium head blight in wheat. Fungal Biol. 116:413-426.

Ross, A. R. S., Ambrose, S. R., Cutler, A. J., Feurtado, J. A., Kermode, A. R., Nelson, K., Zhou, R., and Abrams, S. R. 2004. Determination of endogenous and supplied deuterated abscisic acid in plant tissues by high performance liquid chromatography-electrospray ionization tandem mass spectrometry with multiple reaction monitoring. Anal. Biochem. 329:324-333.

Seong, K., Hou, Z., Tracy, M., Kistler, H. C., and Xu, J. R. 2005. Random insertional mutagenesis identifies gene association with virulence in the wheat scab fungus Fusarium graminearum. Phytopathology 95:744-750.

Siewers, V., Kokkelink, L., Smedsgaard, J., and Tudzynski, P. 2006. Identification of an abscisic acid gene cluster in the grey mold Botrytis cinerea. Appl. Environ. Microbiol. 72:4619-4626.

Stephens, A. E., Gardiner, D. M., White, R. G., Munn, A. L., and Manners, J. M. 2008. Phases of infection and gene expression of Fusarium graminearum during crown rot disease of wheat. Mol. Plant-Microbe Interact. 21: 1571-1581.

Sun, Y., Xiao, J., Jia, X., Ke, P., He, L., Cao, A., Wang, H., Wu, Y., Gao, X., Wang, X. The role of wheat jasmonic acid and ethylene pathways in response to Fusarium graminearum infection. Plant Growth Regul. In press. doi:10.1007/s10725-016-0147-1

Takeuchi, J., Okamoto, M., Okiyama, T., Muto, T., Yajima, S., Sue, M., Seo, M., Kanno, Y., Kamo, T., Endo, A., Nambara, E., Hirai, N., Ohnishi, T., Culter, S., and Todoroki, Y. 2014. Designed abscisic acid analogs as antagonists of PYL-PP2C receptor interactions. Nat. Chem. Biol. 10:477-482.

Tanaka, N., Matsuoka, M., Kitano, H., Asano, T., Kaku, H., and Komatsu, S. 2006. Gid1, a gibberellin-insensitive dwarf mutant, shows altered regulation of probenazole-inducible protein (PBZ1) in response to cold stress and pathogen attack. Plant Cell Environ. 29:619-631.

Trapnell, C., Williams, B. A., Pertea, G., Mortazavi, A., Kwan, G., van Baren, M. J., Salzberg, S. L., Wold, B. J., and Pachter, L. 2010. Transcript assembly and quantification by RNA-seq reveals unannotated transcripts and isoform switching during cell differentiation. Nat. Biotechnol. 28:511-515.

Untergasser, A., Cutcutache, I., Koressaar, T., Ye, J., Faircloth, B. C., Remm, M., and Rozen, S. G. 2012. Primer3-New capabilities and interfaces. Nucleic Acids Res. 40:e115.

Vanden Bossche, H., Marichal, P., Gorrens, J., Coene, M. C., Willemsens, G., Bellens, D., Roels, I., Moereels, H., and Janssen, P. A. 1989. Biochemical approaches to selective antifungal activity. Focus on azole antifungals. Mycoses 32:35-52.

Yabuta, Y. 1935. Biochemistry of the 'bakanae' fungus of rice. Agric. Hortic. 10:17-22.

Yamaguchi, S. 2008. Gibberellin metabolism and its regulation. Annu. Rev. Plant Biol. 59:225-251.

Yang, Y.-X., Golam, J. A., Wu, C., Fan, S.-Y., and Zhou, Y.-H. 2015. Crosstalk among jasmonate, salicylate, and ethylene signaling pathways in plant disease and immune responses. Curr. Protein Pept. Sci. 16:450-461.

Zaharia, L. I., Galka, M. M., Ambrose, S. J., and Abrams, S. R. 2005. Preparation of deuterated abscisic acid metabolites for use in mass spectrometry and feeding studies. J. Labelled Compounds Radiopharm. 48:435-445. 